\title{
Toxina botulínica para hipertrofia do masseter
}

\author{
Botulinic toxin for masseter hypertrophy \\ Toxina botulínica para la hipertrofia de masetero
}

Recebido: 04/10/2021 | Revisado: 13/10/2021 | Aceito: 20/10/2021 | Publicado: 22/10/2021

Maria Eduarda Silva Santos

ORCID: https://orcid.org/0000-0002-8015-9200

Faculdade Patos de Minas, Brasil

E-mail: mariahsilvasantoss@gmail.com

Thaís Anicesio dos Santos

ORCID: https://orcid.org/0000-0002-1019-4107

Faculdade Patos de Minas, Brasil

E-mail: tah_anicesio@hotmail.com

Fernando Nascimento

ORCID: https://orcid.org/0000-0003-3568-3887

Faculdade Patos de Minas, Brasil

E-mail: fernando.nacimento@faculdadepatosdemins.edu.br

Victor da Mota Martins

ORCID: https://orcid.org/0000-0001-6631-6161

Centro Universitário de Patos de Minas, Brasil

E-mail: victortag@hotmail.com

Lia Dietrich

ORCID: https://orcid.org/0000-0001-7887-8591

Universidade Federal dos Vales do Jequitinhonha e Mucuri, Brasil

E-mail: lia.dietrich@ufvjm.edu.br

\begin{abstract}
Resumo
Existem opções distintas da neurotoxina botulínica como A, B, C, D, E, F e G. Os tipos A e B são os mais utilizados na prática odontológica. Eles podem ter um efeito controlado bem conhecido no tecido muscular. A toxina botulínica se tornou um medicamento usado para fins funcionais na odontologia e, recentemente, usado com o intuito de cosmético facial. Sua indicação destaca-se em pacientes com hipertrofia do masseter, entre outras. O presente trabalho acadêmico tem como objetivo descrever, através de uma revisão de literatura, o uso da toxina botulínica nos casos de hipertrofia do masseter. Para tanto, foi utilizado uma pesquisa qualitativa de fonte secundária, descritiva com método dedutivo. O uso da toxina botulínica para tratamento da hipertrofia do masseter vem se destacando cada vez mais por sua aplicação ser bastante segura e eficiente. Porém, estudos que comprovam a importância da sua utilização ainda estão escassos.
\end{abstract}

Palavras-chave: Odontologia; Toxina Botulínica tipo A; Músculo Masseter.

\section{Abstract}

There are different options for botulinum neurotoxin such as A, B, C, D, E, F and G. Types A and B are the most used in dental practice. They can have a well-known controlled effect on muscle tissue. Botulinum toxin has become a medicine used for functional purposes in dentistry and recently used for facial cosmetic purposes. Its indication stands out in patients with masseter hypertrophy, among others. This academic work aims to describe, through a literature review, the use of botulinum toxin in cases of masseter hypertrophy. For that, a qualitative research of secondary source, descriptive with deductive method was used. The use of botulinum toxin for the treatment of masseter hypertrophy has been increasingly highlighted, as its application is very safe and efficient. However, studies that prove the importance of its use are still scarce.

Keywords: Dentistry; Botulinum toxin type A; Masseter muscle.

\section{Resumen}

Existen diferentes opciones de neurotoxina botulínica como A, B, C, D, E, F y G. Los tipos A y B son los más utilizados en la práctica odontológica. Pueden tener un efecto controlado bien conocido sobre el tejido muscular. La toxina botulínica se ha convertido en un medicamento utilizado con fines funcionales en odontología y recientemente utilizado con fines cosméticos faciales. Destaca su indicación en pacientes con hipertrofia masetera, entre otros. Este trabajo académico tiene como objetivo describir, a través de una revisión de la literatura, el uso de la toxina botulínica en casos de hipertrofia masetero. Para ello se utilizó una investigación cualitativa de fuente secundaria, descriptiva con método deductivo. El uso de la toxina botulínica para el tratamiento de la hipertrofia de maseteros se ha destacado 
cada vez más, ya que su aplicación es muy segura y eficaz. Sin embargo, aún son escasos los estudios que demuestren la importancia de su uso.

Palabras clave: Odontología; Toxina botulínica tipo A; Músculo masetero.

\section{Introdução}

O músculo masseter faz parte dos músculos do processo mastigatório, podendo existir patogenias relacionadas ao mesmo, sendo uma das mais conhecidas a "hipertrofia do masseter" (Francisco \& Lopes, 2018). Pode apresentar-se uni ou bilateral e sua etiologia é idiopática; porém, alguns autores destacam grande parte de casos relacionados a hábitos parafuncionais, além de não ter predileção por sexo (Ferreira, 2019; Simão, Borba, Silva, \& Almeida, 2014).

O tratamento consiste em técnicas conservadoras e invasivas, sendo de extrema importância resultados estéticos e funcionais eficazes (Vieira, 2010). A toxina botulínica tipo A, por ser uma neurotoxina enérgica, é produzida pelo Clostridium botulinium, e quando infiltrada no músculo, há um bloqueio da acetilcolina, inibindo sua liberação (Francisco \& Lopes, 2018).

O uso da toxina botulínica para hipertrofia do masseter possui grande relevância nos dias atuais. Alguns pacientes apresentam assimetria na face, ou excesso, causando desconforto no contorno do rosto dos mesmos. Essa terapia conservadora vem sendo usada para obtenção de um contorno facial mais atenuado e harmonioso. Por não ser uma técnica invasiva, ela torna-se bastante acessível (Simão et al., 2014).

O título "Toxina Botulínica para hipertrofia do Masseter", surgiu diante do estudo sobre o tema, que se revela interessante e polêmico, por substituir técnicas cirúrgicas. Neste trabalho, busca-se propor sobre a importância da toxina botulínica e da implementação das suas técnicas que podem ser utilizadas tanto para fins estéticos, quanto funcionais. Assim, dentre as diversas formas de aplicabilidade, tem-se observado um método simples e seguro com resultados clínicos satisfatórios para os pacientes. Daí a importância do estudo sobre o tema, para aprimoramento da técnica. O objetivo deste trabalho é descrever, através de uma revisão de literatura, o uso da toxina botulínica nos casos de hipertrofia do masseter.

\section{Metodologia}

A metodologia do presente trabalho consiste em uma revisão de literatura compilada: de materiais publicados (incluindo principalmente livros, artigos de periódicos e materiais atualmente disponíveis na Internet).

Caracteriza-se do tipo descritiva pois está relacionada à descrição de fatos ou fenômenos. Esse tipo de pesquisa é mais aprofundado do que a pesquisa exploratória. Entre os alunos, é comum confundir pesquisa descritiva com pesquisa explicativa, porque na verdade ambas são preocupações comuns com variáveis relacionadas. Mas a semelhança acaba aqui, porque o estudo de personagens descritivos vai além da mera exploração, mas ainda não chegou ao ponto de explicar um dado fenômeno (Kauark, Manhães, \& Medeiros, 2010).

Para isto, utilizou-se a pesquisa qualitativa. A natureza da pesquisa qualitativa requer um estudo aprofundado do contexto e do local em que é realizada, bem como da interação entre o pesquisador e o sujeito. O olhar indiferente do observador não será capaz de captar muitas informações disponíveis. Os pesquisadores estarão em processo de pesquisa imersiva, em comparação com outros tipos de pesquisa; os estudos qualitativos costumam ter um número maior de variáveis. Em princípio, na pesquisa qualitativa, uma grande quantidade de informações não pode ser excluída desde o início, o que dificulta a determinação efetiva da causa de um determinado fenômeno sem a necessidade de persuasão por meio da argumentação, como fazem os pesquisadores. Portanto, a pesquisa qualitativa mostrou-se a mais adequada, pois a qualidade dos dados é mais importante do que a quantidade de dados. Além das características subjetivas, são utilizadas narrativas escritas (Rispoli et al., 2008).

As fontes secundárias são informações suficientes e totalmente verificadas e referenciadas à fonte primária. Portanto, 
são fontes onde as pessoas podem buscar os mais diversos resultados de dados ou inferências originalmente apontadas por terceiros. Essa fonte foi escolhida porque o autor comparou e analisou os dados, revisou os resultados deste artigo e tirou conclusões dos artigos e livros (Nascimento et al., 2012).

O método adotado foi o dedutivo: busca de leis gerais no ramo da ciência. A lógica dedutiva começa com um método abrangente de fazer propostas específicas por proposições. O método de inferência é acusado de ser desfavorável à ciência por se basear em proposições gerais fáceis de entender e reparáveis. Em outras palavras, a partir de uma verdadeira compreensão dos fatos e fenômenos, só podemos tirar as mesmas conclusões. No entanto, isso é incorreto, porque os casos de bom senso não só podem nos ajudar a entender a causa, mas também podem estar relacionados a situações específicas e nos preparar para responder (Kauark et al., 2010, Lamme, 2011).

Para realização deste trabalho foi realizada uma pesquisa sobre a toxina botulínica para hipertrofia do masseter, a base de dados foi SCIELO e BVS. Os descritores usados na busca foram palavras em português na base de dados pesquisada. Nessa seleção foram utilizados descritores "hipertrofia do masseter", "toxina botulínica", "toxina botulínica na odontologia", "toxina botulínica para hipertrofia do masseter" e "músculo masseter", sendo encontrados 19 resultados relacionados aos termos citados. Os artigos são referentes aos anos de 2000 a 2019.

\section{Músculo Masseter e Funcionalidade}

O músculo masseter, quadrilateral e forte, é recoberto pela fáscia massetérica. Encontra-se localizado ao lado do ramo da mandíbula, sendo considerado valioso para uma harmonia facial (Vieira, 2010).

É fundamental para uma mastigação apropriada. Ele pode ser delimitado em parte superficial e parte profunda. A parte superficial apresenta-se maioral e germina da parte inferior do arco zigomático, abrangendo a metade do arco zigomático. Apresenta-se mais anteriormente. A parte profunda apresenta-se menor e é originada da margem inferior e da face medial do arco zigomático, abrangendo-se até o limite da eminência articular (Francisco \& Lopes, 2018).

O osso da mandíbula é influenciado pela ação dos músculos da mastigação e produz uma variedade de movimentos (levantar, abaixar, estender, retrair, movimento lateral). A ascensão mandibular é também realizada pelo masseter, além do temporal anterior e pterigóideo medial. Depois que as fibras musculares do músculo masseter se contraem, elas projetam o osso mandibular para cima para promover o contato entre as arcadas dentárias (Vieira, 2010).

\subsection{Hipertrofia do masseter: patologia e diagnóstico}

A hipertrofia do músculo masseter é uma situação rara, benigna, que consiste em um desenvolvimento demasiado, unilateral ou bilateral do músculo, alterando o contorno facial, causando um incômodo estético e prejuízos psicossociais no indivíduo (Manganello-Souza, Oliveira, Alpire, \& Trigo-Merida, 2000).

Esse aumento excessivo pode alterar a função do músculo, causando trismo, protrusão e bruxismo, cefaleia e tensão muscular (Vieira, 2010, Pinzon et al., 2018).

Pode estar associado com a hipertrofia de outros músculos da mastigação como o músculo temporal, podendo acometer o temporal unilateralmente ou bilateralmente (Francisco \& Lopes, 2018).

Para um diagnóstico clínico preciso, é imprescindível uma complementação com exames de imagens: radiografia, ultrassonografia e ressonância magnética. Clinicamente, é necessário que o paciente aperte os dentes em máxima intercuspidação, para que ao tocá-lo, possa identificar o aumento excessivo do tônus muscular e também examinar anormalidades no formato do ângulo da mandíbula (Manganello-Souza et al., 2000).

É importante ressaltar que a hipertrofia do masseter pode assemelhar-se a outras patologias (Vieira, 2010).

Inúmeros autores relatam que o diagnóstico diferencial pode ser realizado de outras alterações da glândula parótida e 
do músculo masseter, envolvendo parotidite, tumefações malignas ou benignas da glândula parótida, adipoma, tumor dos vasos ou músculos, e tumores ósseos mandibulares (Pinzon et al., 2018).

\subsubsection{Fatores etiológicos}

A hipertrofia do masseter é uma anomalia de caráter incógnito. Pode ser congênita ou adquirida, sendo a última a mais comum. Essa pode estar relacionada com diversas disfunções: ausência dentária, maus hábitos mastigatórios, apertamento dentário, consumo frequente de chicletes de goma, problemas na ATM, atritar os dentes enquanto dorme como hábitos e invenções atuais de uso de dispositivos para exercitar a região e criar tal hipertrofia. Com isso, pacientes que possuem esses costumes frequentes podem desencadear problemas na musculatura massetérica, desenvolvendo a hipertrofia. Outro fator que pode ser uma das grandes causas é o desequilíbrio psicológico, estimulando uma modificação no estado de tensão elástica do masseter (Vieira, 2010).

Comumente atinge adultos entre o $2^{\circ}$ e $3^{\circ}$ decênio de vida; não há propensão em relação ao sexo. Pode-se manifestar em seres de todos os grupos, porém, em indivíduos de ascendência asiática podem ser mais habituais (Pinzon et al., 2018).

\subsubsection{Possibilidades de tratamento em hipertrofias acentuadas}

Há distintas possibilidades como tratamento da hipertrofia do masseter; com isso pode-se classificar em um método mais invasivo, o cirúrgico e o método menos invasivo, o não cirúrgico. Na literatura há diversas técnicas cirúrgicas empregadas para tratamento da hipertrofia (Agrawal, Singh, Shivamurthy, \& Varghese, 2011; Pinzon et al., 2018; Vieira, 2010).

A modalidade cirúrgica foi sugerida por Gurny pela primeira vez no ano de 1947. Seu método baseia-se realizando uma incisão submandibular e a retirada de 3/4 a 22/3 de volume na musculatura. Já em 1950 Adams sugeriu uma osteotomia na região do ângulo da mandíbula; nessa mesma época Martensson realizou em uma paciente que apresentava apertamento dentário e hipertrofia unilateral a retirada da inserção do masseter, fazendo uma incisão triangular. Em 1977 Beckers tratou de 17 pacientes utilizando o método intraoral, o qual retirava do masseter hipertrofiado uma tira muscular interna, havendo assim uma diminuição da cicatriz do rosto. Wood em 1982, sugeriu um meio ao qual não removia nenhuma parte do masseter (Agrawal et al., 2011; Vieira, 2010; Oliveira, Nogueira, Vasconcellos, \& Vasconcellos, 2004).

$\mathrm{Na}$ atualidade a técnica cirúrgica pode ser executada tanto por via intrabucal quanto por extrabucal. Porém, a extrabucal tem maior indicação por apresentar uma melhor visibilidade no ato cirúrgico; mas essa técnica pode ocasionar danos ao nervo facial e trazer problemas estéticos como cicatriz (Pinzon et al., 2018).

Com a modernidade, novas ferramentas de trabalho e habilidades cirúrgicas surgiram como: instrumentos giratórios, afastadores e até mesmo um endoscópio intrabucal, o que acabou beneficiando a técnica intrabucal. Além do mais ela tem benefícios comparados à extrabucal, como uma melhor aparência estética, ou seja, não deixa cicatrizes e reduz o risco de acometer os nervos da mandíbula; com isso ela é a mais usada atualmente (Agrawal et al., 2011; Pinzon et al., 2018; Vieira, 2010).

Já o tratamento com método tradicionalista não cirúrgico abrange diversas opções de tratamento como: tratamento psicológico para pacientes que possuem distúrbios psiquiátricos, uso de fármacos que acalmam ou neutralizam contrações involuntárias de músculos, ansiolíticos, uso de medicamentos que relaxam a tensão muscular, modificações de dentes caso necessário, correção de contato prematuro, eliminação de hábitos involuntários ou inconscientes do paciente (Oliveira et al., 2004; Pinzon et al., 2018; Vieira, 2010).

Como destaque nos tratamentos tradicionalistas que acabam sendo um tratamento conservador em relação às técnicas cirúrgicas, pode-se ressaltar a toxina botulínica A como uma boa opção; um método que vem sendo bastante utilizado pelos 
profissionais até mesmo substituindo as opções que existem (Oliveira et al., 2004; Pinzon et al., 2018).

Em 1994, Smyth, Moore, Wood, fizeram a primeira aplicação de toxina botulínica no músculo masseter onde o paciente apresentava um quadro de hipertrofia (Oliveira et al., 2004). A toxina botulínica age inibindo o mediador químico acetilcolina; com isso reduz as contrações musculares e, consequentemente, o músculo sofre uma atrofia (Pinzon et al., 2018, Oliveira et al., 2004).

Esse tipo de tratamento não é definitivo; com isso o paciente, em torno de 6 meses, dependendo a quantidade da dose aplicada, deve voltar ao profissional para fazer sua reaplicação (Oliveira et al., 2004; Pinzon et al., 2018,).

\section{Toxina Botulínica}

A toxina botulínica, descoberta por Justinus Kerner, no ano de 1822, tem sido usada com grande destaque na Odontologia nos dias atuais para fins estéticos e funcionais (Vasconcelos, Sousa Filho, Cavalcante, \& Vasconcelos, 2017). As neurotoxinas botulínicas são bactérias anaeróbias, originadas da Clostridium botulinium, sendo consideradas de alta toxicidade (14). Elas impedem a liberação da acetilcolina, tendo como efeito a paralisação do músculo. Encontram-se 7 sorotipos de TxB, sendo eles: A, B C, D, E, F e G. Destaca-se o tipo A, comumente utilizado em fins estéticos, possuindo maior potência se comparado aos demais. No comércio, encontram-se diversas marcas para toxina botulínica A, no entanto, as marcas mais conhecidas são: BOTOX, DYSPORT, XEOMIN e PROSIGNE (Vasconcelos et al., 2017). No Brasil, a sua aprovação para procedimentos estéticos foi em 2000 (Shimaoka, Andrade, \& Carvalho, 2011). Em 2014, o Conselho Federal de Odontologia (CFO), liberou a utilização da toxina botulínica em meios odontológicos, e indeferiu sua aplicabilidade em meios não odontológicos (Barbosa \& Barbosa, 2017).

\subsection{Mecanismo de ação}

A toxina botulínica impede a liberação de neurotransmissores da acetilcolina nas células nervosas, diminuindo a constrição do músculo (Sposito, 2009).

A TxB possui dois tipos de cadeia: leve e pesada, que se divergem em $\mathrm{HC}$ e $\mathrm{HN}$, e são consideradas peptídicas simples. O HC associa-se aos neurônios motores superiores, já o HN tem a função de incorporação e propagação do impulso nervoso por meio de células nervosas. A ação ativa da toxina botulínica na célula é realizada pela cadeia leve. Essa molécula irá disputar com a acetilcolina, que tem como responsabilidade causar a apreensão muscular, impedindo sua ação. A TB anula as proteínas da acetilcolina, bloqueando contato com as vesículas transmissoras que contêm os neurotransmissores. Com isso, a neurotoxina terá conexão com a fenda pré-sináptica, havendo um relaxamento muscular (Vasconcelos et al., 2017).

No músculo estriado esquelético, seu efeito pode ter, em média, de 2 a 5 dias, podendo haver exceções, e seu efeito se expandir em até duas semanas. Assim que introduzido, seu efeito pode durar de 1 a 6 meses, sendo considerada uma média de 3 a 4 meses. Na sua fase mais acentuada, pode-se notar atrofiação muscular e modificação das fibras, através de análise histológicas. Depois de 2 a 3 meses, sua eficácia reduz gradativamente (Colhado, Boeing, \& Ortega, 2019).

\subsection{Funcionalidade}

A toxina botulínica pode exercer várias funções, tanto para fins estéticos e/ou funcionais (Francisco \& Lopes, 2018). Embora inúmeras designações para aplicação terapêutica da TxB sejam indicadas para síndromes neurológicas, que podem ser constatados excessos de movimentos ou falta dos mesmos, a sua utilidade tem sido bastante ampliada na atuação farmacológica, abrangendo desordens oftalmológicas, ortopédicas, urológicas, dermatológicas, gastrointestinais, secretórias e cosméticas (Shimaoka et al., 2011). Outro importante papel da sua atuação pode ser no alívio da dor, impedindo os neuropeptídios relacionados à sensação dolorosa que ativam o sistema nervoso central. Na década de 1970, a toxina botulínica 
foi considerada como um fator terapêutico para correção do estrabismo. A partir daí sua aplicabilidade tem-se amplificado em diversas áreas (Shimaoka et al., 2011).

No Brasil, em acessão com a Lei Federal n $n^{\circ}$ 5.081/166, o Cirurgião-Dentista pode usufruir da BoNT para fins específicos da Odontologia (Francisco \& Lopes, 2018). Introduções da toxina botulínica são eficazes para distintas disfunções musculares. Além do mais, quando injetada nos tecidos epiteliais glandulares, ela age restringindo a liberação das secreções. Sendo assim, o CD pode atuar em casos de hipertrofia do masseter, excesso de salivação, erupção passiva alterada, bruxismo, sorriso assimétrico, disfunção temporomandibular (DTM), trismo e, em alguns casos pode-se fazer a aplicação nos músculos masseter e temporal para diminuição da força dos mesmos quando submetidos a implantes de carga imediata. Por fim, quando se trata do quesito estético, a TxB possui alta relevância, trazendo equilíbrio e traços mais definidos para o rosto e, consequentemente, uma melhor harmonia facial (Shimaoka et al., 2011).

\subsection{Vantagens e limitações}

A toxina botulínica nos surpreende positivamente pelo fato de o paciente voltar às suas atividades diárias de imediato, não necessitando se submeter à hospitalização, além de obter um efeito idôneo, melhorando seu bem estar (Francisco \& Lopes, 2018). Existe também uma redução de efeitos adversos e da ingestão de fármacos adjuvantes, não sendo necessário o uso contínuo de outros medicamentos (Colhado et al., 2019).

A TxB tem sido usada como forma de tratamento em diversas áreas, por exemplo: na oftalmologia, em casos de estrabismos e blefaroespasmos essenciais benignos; nas distonias focais, em casos de contrações musculares involuntárias; na hiperidrose, em casos de sudorese intensa; na cefaleia, havendo redução na duração da dor de cabeça, frequência e intensidade; na urologia, em casos de bexiga hiperativa, e também tem sido eficaz no tratamento contra a BPH (hiperplasia benigna da próstata), sem alterar a função sexual. A TxB também está presente na área funcional e estética, atuando na região orofacial. No que se diz respeito à parte funcional, ela se mostra eficiente nos quadros de hipertrofia do masseter, DTM, sialorreia, dores orofaciais, bruxismo, sorriso assimétrico, quielite angular, e após a realização de procedimentos de cirurgias periodontais e implantes dentários. Quanto à estética, a toxina juntamente com outras categorias estéticas, age como preenchedores de tecidos moles, laser, estimulador de colágeno, e reduz às linhas de expressão, trazendo uma maior harmonia a face do paciente (Marcino, Aguiar, Vieira, \& Magalhães, 2014).

A toxina botulínica é contraindicada nas seguintes situações: deve ser abdicada em grávidas, pelo fato da desinformação ao que se pode causar ao feto, em pessoas que possuem transtornos mentais, e também em pacientes com hipersensibilidade. Pacientes que possuem alergia aos nutrientes presentes no ovo também são contraindicados. Associações com alguns medicamentos, como ciclosporinas, possuem restrição ao uso da toxina (Barbosa \& Barbosa, 2017).

Quando aplicada de forma inadequada, pode trazer malefícios à face do paciente, causando assimetria, dor, edema, vermelhidão, redução dos movimentos corporais, mudança de voz, boca seca e obtendo por fim, resultados indesejados. Por isso, o profissional deve ter um alto conhecimento sobre a anatomia do rosto, aprimorando suas técnicas e conhecendo pontos de aplicações permitidos e não permitidos, visando uma maior segurança do paciente (Shimaoka et al., 2011, Marcino et al., 2014).

\subsection{Técnica de aplicação}

A toxina botulínica serve como um tratamento paliativo nos problemas de DTM (disfunção temporomandibular), hipertrofia do masseter, entre outros, é feita a aplicação nos músculos masseter e temporal, causando uma redução do tônus muscular no paciente. Também reduz a sensibilidade à dor. No músculo masseter, a região é dividida em 6 porções, três na parte inferior e três na parte superior. Sendo que, as aplicações devem ser feitas no terço inferior. Na área inferior, deve-se 
delimitar três pontos, havendo um distanciamento de 2 espaço $\mathrm{cm}$. A toxina penetra nos tecidos em uma região de 1,5 a 3 espaço $\mathrm{cm}$ em volta da área aplicada. Não é viável a aplicação da TxB no terço superior, por trazer danos à glândula parótida e ductos. Não há uma norma na dose a ser aplicada, portanto, cada paciente possui sua particularidade e deve ser avaliado especificamente. É importante ressaltar que, pacientes que fazem o uso de óculos diariamente deve ser aplicada uma quantidade maior da toxina, por possuírem uma maior movimentação muscular da face (Pedron, 2016; Vasconcelos et al., 2017).

Para sua aplicação faz-se necessário o uso de agulha de insulina. Para aliviar o desconforto com a ruptura da pele com a introdução da agulha, utiliza-se compressa de gelo, ou anestésico tópico local, como a prilocaína. Para a dissolução, deve-se obedecer as seguintes etapas:

1 - deve-se utilizar uma agulha apropriada para reconstrução e puxar a dose certa de diluente, sendo em média 2 ou 4 $\mathrm{ml}$, e soro fisiológico $0,9 \%$.

2 - Introduzir a agulha instruindo em um ângulo de $45^{\circ}$, inserindo com cautela o diluente no frasco.

3 - Virar o frasco devagar para obter homogeneização.

4 - Colocar a agulha contendo a toxina botulínica diluída no frasco, difundindo na seringa de insulina para realizar a aplicação. No exemplo a seguir, consideramos $4 \mathrm{ml}$ de diluição:

$4 \mathrm{ml}$ da solução diluída no frasco equivale a $100 \mathrm{U} 0,1 \mathrm{ml}$ equivale a $\mathrm{X}$, aplica-se regra de 3 . $\mathrm{X}=2,5 \mathrm{U}$, significa dizer que a cada $0,1 \mathrm{ml}$ se aplica $2,5 \mathrm{U}$ (Vasconcelos et al., 2017).

\section{Discussão}

A Hipertrofia do Masseter é uma etiopatogenia pouco conhecida, porém, vários casos têm sido descritos na literatura nos últimos anos. Ainda não foi descoberta uma causa específica para tal, mas, existem situações que podem estar associadas com o seu surgimento, sendo: bruxismo, esforços mastigatórios, doenças mentais e, modificações da ATM (Rispoli et al., 2008).

Apresenta-se edema no ângulo mandibular, interferindo negativamente no contorno facial do paciente e, consequentemente, na sua estética. Alguns autores afirmam que pode haver sintomatologia dolorosa, enquanto outros não defendem essa teoria, afirmando não ser comum. Por haver confusões entre resultados diagnosticados com patologias semelhantes à Hipertrofia do masseter, é de suma importância um diagnóstico correto e preciso (Ênia et al., 2021; Simão et al., 2014).

$\mathrm{Na}$ literatura encontram-se formas de tratamento para a Hipertrofia do Masseter, sendo cirúrgico ou conservador. Existem diversas técnicas cirúrgicas como tratamento da mesma, porém, nos dias atuais, não são mais preconizadas por trazer desconfortos e resultados não tão satisfatórios esteticamente para o paciente. Por isso, a técnica conservadora vem tomando espaço para essa reabilitação, destacando a aplicação da toxina botulínica. É uma neurotoxina potente, e possui 7 tipos: A ao G, sendo o tipo A o mais utilizado para esse tratamento. Tem como função o bloqueio reversível do músculo, agindo em um período de 4 a 6 meses. Ela apresenta vantagens por ser não invasiva, trazer resultados estéticos satisfatórios, ser introduzida em poucas sessões, apresentar baixos índices de contraindicações e grande margem de segurança, e logo após a sua aplicação o paciente pode executar suas atividades rotineiras normalmente, além de ser bastante utilizada no meio odontológico para fins estéticos e funcionais (Marcino, Silveira, Raulino Neto, Martins, \& Pessoa, 2017).

\section{Considerações Finais}

A Hipertrofia do Masseter é uma anomalia que ocasiona problemas estéticos e distúrbios mentais e sociais ao 
paciente. Existem diversas formas de tratamento, porém, destaca-se o uso da TxB, por ser uma técnica conservadora, trazendo mais vantagens, e sendo bastante eficiente. Devido ao grande destaque que a toxina botulínica vem alcançando, ela tem sido utilizada para vários fins, tanto funcionais, quanto estéticos.

Por fim, é de suma importância novas pesquisas para enfatizar o uso da Toxina Botulínica como tratamento para diversas etiopatogenias, incluindo a Hipertrofia do Masseter.

\section{Referências}

Agrawal, G., Singh, S., Shivamurthy, D., \& Varghese, D. Tratamento cirúrgico da hipertrofia massetérica e retrognaismo mandibular. Nat Journ of Maxillofa Surg, 2(1), 96-99.

Barbosa, C. M. R., Barbosa, J. R. A. (2017). Toxina Botulínica em Odontologia. Rio de Janeiro: Elsevier.

Colhado, O. C. G., Boeing, M., Ortega, L. B. (2019). Toxina botulínica no tratamento da dor. Rev Bras Anestesiol, 59(3), 366-381.

Ferreira, G. M. (2019). Toxina botulínica: efeitos indesejados nos músculos mastigatórios. Rev face magazine. https://facemagazine.com.br/efeitosindesejados-da-toxina-botulinica-nos-musculos-mastigatorios/

Francisco, J., Lopes S. (2018). Hipertrofia benigna do masseter: tratamento por toxina botulínica [Trabalho de conclusão de curso de graduação em Odontologia] Faculdade de Ciências da Saúde, Brasília.

Kauark, F. S., Manhães, F. C., \& Medeiros, C. H. (2010). Metodologia da pesquisa: um guia prático. Bahia: Via litterarum.

Lamme, M. (2011). Metodologia da Pesquisa Jurídica: técnicas de investigação, argumentação e redação. São Paulo: Elsevier.

Manganello-Souza, L., Oliveira, A., Alpire, M., \& Trigo-Merida, J. (2000). Hipertrofia do músculo masseter. Rev bras cir plást. 15(1), 51-54.

Marcino, A., Aguiar, U., Vieira, P. G. M., \& Magalhães, S. R. (2014). Toxina botulínica e sua aplicação na odontologia [Trabalho de conclusão de curso de graduação em Odontologia]. Universidade Vale do Rio Verde, Belo Horizonte.

Nascimento, G. K. B. O., Cunha, D. A., Lima, L. M., Moraes, K. J. R., Pernambuco, L. A., Régis, R. M. F. L. et al. (2012). Eletromiografia de superfície do músculo masseter durante a mastigação: uma revisão sistemática. Revista CEFAC. 14(4), 725-731.

Oliveira, D. V., Nogueira, R. V. B., Vasconcellos, R. J. H., \& Vasconcellos B. C. E. (2004). Hipertrofia do masseter: relato de caso. Revi de Cirur e Traumat Buco-Maxil-Faci; 4(1), 31-38.

Pedron, I. G. (2016). Toxina botulínica: aplicações em odontologia. Santa Catarina; Ponto.

Pinzon, N. A., Rodriguez, E. A. S., Goulart, D. R., Orlate, S., Farah, G. J., Filho, L. I. (2018). Toxina botulínica para o tratamento de distúrbios temporomandibulares. Int. J. Odontostomat. 12(2), 103-109.

Rispoli, D. Z., Camargo, P. M., Pires, Jr. J. L., Fonseca, V. R., Mandelli, K. K., \& Pereira, M. A. C. (2008). Hipertrofia benigna do músculo masseter. Rev bras otorrinolaringol. 74(5), 790-93.

Shimaoka, A. M., Andrade, A. P., \& Carvalho, R. C. R. (2011). O uso da toxina botulínica na odontologia. (5), 1-3.

Simão, N. R., Borba A. M., Silva, A. L. F., \& Almeida N. S. (2014). Hipertrofia benigna do músculo masseter - relato de caso. Rev. Assoc. Paul Cir. 68 (4), $351-5$.

Sposito, M. M. M. (2009). Toxina Botulínica do Tipo A: mecanismo de ação. Acta fisiat 16 (1), 25-37.

Vasconcelos, A. F. M., Filho, G. C. S., Cavalcante, A. B, \& Vasconcelos, B. C. E. (2017). Toxina botulínica nos músculos masseter e temporal: considerações farmacológicas, anatômicas e clínicas. Rev. Cir. Traumatol Buco-Maxilo-Facial. 16(2), 50-54.

Vieira, J. G. (2010). Metodologia de pesquisa científica na prática: Metodologia de pesquisa. Curitiba: Fael.

Ênia, J. R. N., Fernandes, J. G. A., Nascimento, F., Silva, L. A. M., Reis, T., \& Dietrich, L. (2021). Toxina botulínica no tratamento da paralisia facial: um tratamento de reabilitação minimamente invasivo. Pesquisa, Sociedade e Desenvolvimento, 10(5), e40510515204.

Martins, R. R., Silveira, A. M. M., Raulino Neto, J. S., Martins, J. C. G., \& Pessoa, C. V. (2017). Toxina botulínica tipo A no tratamento de rugas: uma revisão de literatura. Mostr Cient Farm, 3(1), 1-4. 